\title{
70. SULFIDES IN THE CORE FROM LEG 37 DRILL HOLES
}

\author{
W.H. MacLean, Department of Geological Sciences, McGill University, Montreal, Canada
}

\section{INTRODUCTION}

There are three types of sulfide occurrences in the igneous rocks cored in the drill holes of Leg 37: (1) rounded "globules" and interstitial polymineralic grains composed of $\mathrm{Fe}-\mathrm{Cu}-\mathrm{Ni}-\mathrm{Co}$ sulfides and occasional magnetite, (2) individual sulfide grains of chalcopyrite, pyrrhotite, pentlandite, bornite, and pyrite within the rocks, and (3) pyrite grains on fracture surfaces, in veinlets, and in vesicles. Altogether, sulfides comprise less than $0.5 \%$ of the rock and in the basalt at least $99 \%$ of the sulfide is pyrite belonging to type (3). This pyrite is distributed so erratically that a precise estimate of its content is difficult to make. It is, however, readily distinguished from the other types of sulfide mineralization.

\section{ANALYSIS}

The sulfides were studied on polished thin sections, and all types of sulfides were analyzed for $\mathrm{Fe}, \mathrm{Co}, \mathrm{Ni}$, $\mathrm{Cu}, \mathrm{Zn}, \mathrm{Mn}, \mathrm{As}$, and $\mathrm{S}$ with an electron microprobe. $\mathrm{Zn}, \mathrm{Mn}$, and usually As were below detection limits $(0.02 \%)$. Because most globules were less than $4 \mu \mathrm{m}$ in diameter, the electron beam sometimes overlapped on surrounding silicate minerals or glass, resulting in low totals for analyses. Magnetite is also present in globules but oxygen was not calculated. Individual minerals were analyzed in some globules and frequently only one mineral, usually pyrrhotite, is present. The results of the microprobe analyses are given in Table 1 and summarized in Figures 1-4, which also show the position of the samples in each drill hole and other data.

\section{Globules}

The globules occur in glassy and finely crystalline basalt, in gabbro, and in peridotite. A few globules 8 $\mu \mathrm{m}$ in diameter were located but the majority of them were less than $4 \mu \mathrm{m}$. They make up $0.0001 \%$ or less of the sample where the sample is unaltered and are considered to be magmatic in origin. Bulk chemical analyses of the globules vary widely due to their inherent mineralogical inhomogeneity and small size. But with the large number of samples probed, the average analysis of globules listed below are probably representative.

\begin{tabular}{lccrccc} 
& $\mathrm{Fe}$ & $\mathrm{Cu}$ & $\mathrm{Ni}$ & $\mathrm{Co}$ & $\mathrm{S}$ & $\mathrm{Ni} / \mathrm{Cu}$ \\
\cline { 2 - 7 } Basalt & 50.7 & 6.8 & 2.6 & 0.3 & 33.7 & 0.5 \\
Gabbro & 49.6 & 5.6 & 5.4 & 0.2 & 36.2 & 1.0 \\
Peridotite & 30.0 & 7.8 & 26.0 & 2.8 & 33.5 & 3.3
\end{tabular}

Magnetite ranges from 0 to $25 \%$.

The partition of these transition metals between synthetic sulfide and silicate liquids has been measured ex- perimentally by MacLean and Shimazaki (in preparation). The "metal in sulfide liquid/metal in silicate liquid" coefficients are: $\mathrm{Fe}(1.2), \mathrm{Co}(7), \mathrm{Ni}(150)$, $\mathrm{Cu}(50)$, and $\mathrm{Zn}(0.5)$. These coefficients explain the absence of $\mathrm{Zn}$, the low $\mathrm{Co}$, and the higher amounts of $\mathrm{Ni}$ and $\mathrm{Cu}$ adequately, assuming they were all present in about equal amounts in the magma (see Prinz, 1967). However, the $\mathrm{Ni} / \mathrm{Cu}$ ratio for globules in the basalts is 0.5 , whereas $\mathrm{Ni}$ was probably initially as high as $\mathrm{Cu}$ in the magma. $\mathrm{Ni}$ contents of the magma are, however, controlled largely by olivine fractionation. The formation of the globules may have been late in the crystallization history of the basalt due to loss of sulfur as vapor, thus much $\mathrm{Ni}$ would be tied up in olivine and the $\mathrm{Ni} / \mathrm{Cu}$ ratio of the globules would be low. The $\mathrm{Ni} / \mathrm{Cu}$ ratio for the gabbro is 1.0 , possibly indicating little or no loss of sulfur in the intrusive environment and early globule formation. The higher $\mathrm{Ni} / \mathrm{Cu}$ ratio of 3.3 in the peridotite indicates equilibrium of the globules with a more picritic magma.

The metal contents and low $\mathrm{Ni} / \mathrm{Cu}$ ratios of the globules are similar to those studied in Hawaiian basalts by Desborough et al. (1968). They are also very similar to sulfide liquid extracted from a lava lake in Hawaii by Skinner and Peck (1969). Magmatic sulfide ores associated with gabbroic (noritic) rocks at Sudbury, Ontario, and elsewhere have $\mathrm{Ni} / \mathrm{Cu}$ ratios close to 1 , whereas ores in ultramafic rocks have higher ratios, usually 10:1 or higher (Naldrett, 1973).

\section{Individual Sulfide Grains}

Individual sulfide grains occur in all rock types in all the holes. Chalcopyrite, pyrrhotite, and very minor pyrite form about $0.001 \%$ of basalt that has not been affected by late smectite-calcite alteration. The sulfides form interstitially to silicate grains and are usually less than $20 \mu \mathrm{m}$ in largest dimension. The same minerals are found in the gabbro at Site 334 but are larger and more abundant. Pentlandite and chalcopyrite are plentiful in the serpentinized peridotite, forming about $0.5 \%$ of the rock. A very small amount of bornite was found in the peridotite.

Microprobe analyses show a wide range of compositions, from $\mathrm{CuFeS}_{2}$ to $\mathrm{CuFe}_{2} \mathrm{~S}_{3}$, in the minerals listed as chalcopyrite in the basalts. They probably represent "intermediate solid solution" (Cabri, 1973) formed during the cooling of the rocks. Chalcopyrite in the peridotite and gabbro is close to $\mathrm{CuFeS}_{2}$ in composition.

Pentlandite is the most common individual sulfide in peridotite, and it also occurs in the globules. The $\mathrm{Ni} / \mathrm{Ni}+\mathrm{Fe}$ ratios of pentlandites from this rock are plotted in Figure 4. The two types of pentlandite occurrences are indistinguishable in composition and cluster close to $0.49\left(\mathrm{Fe}_{4.6} \mathrm{Ni}_{4.4} \mathrm{~S}_{8}\right)$, indicating 
TABLE 1

Microprobe Analyses of Sulfide Minerals in Leg 37 Cores

\begin{tabular}{|c|c|c|c|c|c|c|c|c|}
\hline $\begin{array}{l}\text { Pol. } \\
\text { Sect. }\end{array}$ & $\begin{array}{c}\text { Sample } \\
\text { (Interval in } \mathrm{cm} \text { ) }\end{array}$ & $\begin{array}{c}\text { Material } \\
\text { Analyzed }\end{array}$ & $\mathrm{Cu}$ & $\mathrm{Ni}$ & $\mathrm{Fe}$ & $\mathrm{Co}$ & $\mathrm{S}$ & Total \\
\hline \multicolumn{9}{|c|}{ Hole 332A } \\
\hline \multirow{8}{*}{ DAG-1 } & $12-1,93-92$ & $\mathrm{gb}$ & 11.52 & 1.44 & 47.32 & 0.0 & 33.98 & 94.33 \\
\hline & & $\mathrm{gb}$ & 4.53 & 0.59 & 55.22 & 0.08 & 34.60 & 95.05 \\
\hline & & $\mathrm{gb}$ & 1.09 & 3.30 & 56.90 & 0.27 & 35.45 & 97.03 \\
\hline & & $\mathrm{cp}$ & 21.40 & 0.14 & 38.87 & 0.30 & 34.96 & 95.72 \\
\hline & & py & 0.02 & 0.06 & 46.88 & 0.0 & 54.12 & 101.09 \\
\hline & & py & 0.0 & 0.0 & 47.20 & 0.05 & 52.91 & 100.16 \\
\hline & & py & 0.01 & 0.02 & 48.10 & 0.13 & 53.03 & 101.36 \\
\hline & & py & 0.04 & 2.91 & 42.17 & 0.95 & 51.08 & 97.17 \\
\hline \multirow{3}{*}{ DAG-2 } & $26-1,104-108$ & $\mathrm{gb}$ & 10.53 & 6.19 & 41.67 & 0.28 & 32.01 & 91.00 \\
\hline & & $\mathrm{gb}$ & 16.84 & 3.67 & 39.69 & 0.38 & 31.01 & 92.05 \\
\hline & & $\mathrm{gb}$ & 17.54 & 6.88 & 36.56 & 0.37 & 24.07 & 95.92 \\
\hline \multicolumn{9}{|c|}{ Hole 332B } \\
\hline \multirow[t]{6}{*}{ DAG-3 } & $6-3,105$ & $\mathrm{gb}$ & 3.55 & 0.63 & 58.23 & 0.12 & 34.27 & 96.90 \\
\hline & & $\mathrm{gb}$ & 6.14 & 0.42 & 54.51 & 0.04 & 34.22 & 96.46 \\
\hline & & $\mathrm{gb}$ & 0.50 & 0.47 & 58.45 & 0.06 & 35.96 & 95.45 \\
\hline & & $\mathrm{gb}$ & 7.09 & 0.16 & 54.89 & 0.18 & 31.81 & 94.16 \\
\hline & & gb & 9.78 & 0.85 & 52.21 & 0.15 & 36.22 & 99.27 \\
\hline & & $\mathrm{gb}$ & 3.99 & 0.74 & 57.98 & 0.15 & 36.89 & 99.82 \\
\hline \multirow[t]{3}{*}{ DAG-4 } & $9-1,36-39$ & $\mathrm{gb}$ & 9.43 & 10.94 & 36.71 & 0.87 & 31.87 & 89.90 \\
\hline & & $\mathrm{gb}$ & 0.39 & 0.0 & 61.24 & 0.34 & 31.75 & 93.88 \\
\hline & & $\mathrm{gb}$ & 0.25 & 0.0 & 56.27 & 0.19 & 31.71 & 88.55 \\
\hline DAG-5 & $9-1,40-43$ & $\mathrm{gb}$ & 12.47 & 3.82 & 43.38 & 0.32 & 32.57 & 92.78 \\
\hline \multirow[t]{5}{*}{ DAG-6 } & $15-1,5-7$ & $\mathrm{gb}$ & 3.66 & 0.85 & 57.64 & 0.34 & 34.08 & 97.03 \\
\hline & & $\mathrm{gb}$ & 0.74 & 0.26 & 61.0 & 0.19 & 32.06 & 94.68 \\
\hline & & $\mathrm{gb}$ & 18.50 & 1.00 & 42.22 & 0.27 & 34.32 & 96.69 \\
\hline & & $\mathrm{cp}$ & 19.86 & 0.85 & 34.72 & 0.27 & 37.24 & 93.21 \\
\hline & & py & 0.02 & 0.25 & 44.47 & 0.32 & 49.58 & 94.91 \\
\hline \multirow[t]{11}{*}{ DAG-7 } & $30-1,46-48$ & gb & 2.07 & 0.19 & 60.21 & 0.08 & 32.52 & 95.17 \\
\hline & & $\mathrm{gb}$ & 0.18 & 0.14 & 60.56 & 0.0 & 35.47 & 96.72 \\
\hline & & $\mathrm{gb}$ & 0.92 & 0.24 & 53.64 & 0.0 & 33.83 & 88.83 \\
\hline & & $g b$ & 20.43 & 0.34 & 40.50 & 0.0 & 33.85 & 95.36 \\
\hline & & $\mathrm{gb}$ & 12.35 & 0.40 & 44.38 & 0.0 & 33.05 & 90.40 \\
\hline & & $\mathrm{gb}$ & 0.49 & 0.13 & 58.29 & 0.0 & 33.87 & 93.07 \\
\hline & & $\mathrm{gb}$ & 1.78 & 1.57 & 38.37 & 2.0 & 38.15 & 81.95 \\
\hline & & gb & 2.68 & 1.73 & 37.73 & 1.62 & 37.20 & 81.09 \\
\hline & & $\mathrm{cp}$ & 22.98 & 0.0 & 41.51 & 0.25 & 34.90 & 99.99 \\
\hline & & $\mathrm{cp}$ & 24.90 & 0.0 & 39.11 & 0.15 & 35.43 & 99.89 \\
\hline & & $\mathrm{cp}$ & 31.84 & 0.0 & 28.69 & 0.0 & 36.02 & 96.74 \\
\hline DAG-8 & $33-2,113$ & \multicolumn{7}{|c|}{ No sulfides found } \\
\hline \multirow[t]{11}{*}{ DAG-9 } & $43-3,110$ & $\mathrm{gb}$ & 12.84 & 13.33 & 23.99 & 2.62 & 32.36 & 85.25 \\
\hline & & $\mathrm{gb}$ & 17.86 & 3.86 & 39.69 & 0.24 & 35.64 & 97.38 \\
\hline & & $\mathrm{gb}$ & 13.44 & 4.39 & 40.02 & 0.0 & 33.78 & 91.65 \\
\hline & & $\mathrm{gb}$ & 13.99 & 2.32 & 37.45 & 0.11 & 32.68 & 86.59 \\
\hline & & gbp & 5.86 & 21.31 & 18.11 & 3.25 & 18.11 & 79.96 \\
\hline & & gbp & 31.84 & 0.89 & 28.29 & 0.38 & 36.64 & 98.09 \\
\hline & & $\mathrm{cp}$ & 33.78 & 0.09 & 28.71 & 0.05 & 34.66 & 97.34 \\
\hline & & $\mathrm{cp}$ & 31.03 & 0.0 & 26.20 & 0.0 & 33.87 & 91.20 \\
\hline & & cp & 33.99 & 0.22 & 27.92 & 0.09 & 33.61 & 95.87 \\
\hline & & $\mathrm{cp}$ & 33.10 & 0.04 & 27.44 & 0.0 & 32.31 & 92.91 \\
\hline & & py & 0.0 & 0.33 & 47.49 & 0.16 & 52.99 & 101.01 \\
\hline DAG-10 & $44-3,104-106$ & \multicolumn{7}{|c|}{ No sulfides found } \\
\hline DAG-11 & $44-6,23-27$ & $\mathrm{gb}$ & 2.22 & 1.15 & 60.04 & 0.25 & 35.25 & 99.08 \\
\hline & & gbp & 23.25 & 1.48 & 37.66 & 0.40 & 35.44 & 98.29 \\
\hline & & gbp & 0.86 & 0.0 & 60.71 & 0.33 & 35.31 & 97.38 \\
\hline & & $\mathrm{gb}$ & 0.26 & 0.36 & 59.20 & 0.48 & 38.41 & 98.80 \\
\hline & & gb & 6.81 & 1.14 & 52.20 & 0.43 & 33.28 & 93.95 \\
\hline & & $\mathrm{gb}$ & 4.36 & 2.98 & 48.93 & 2.50 & 36.28 & 95.17 \\
\hline & & $\mathrm{gb}$ & 3.30 & 0.62 & 59.05 & 0.47 & 35.84 & 99.48 \\
\hline & & $\mathrm{gb}$ & 0.0 & 0.66 & 58.80 & 0.41 & 39.94 & 99.88 \\
\hline & & $\mathrm{cp}$ & 21.69 & 0.0 & 39.95 & 0.34 & 35.66 & 97.67 \\
\hline & & $\mathrm{cp}$ & 29.55 & 0.0 & 32.72 & 0.33 & 35.86 & 98.50 \\
\hline & & $\mathrm{cp}$ & 21.94 & 0.0 & 40.13 & 0.38 & 35.34 & 97.80 \\
\hline & & $\mathrm{cp}$ & 22.70 & 0.0 & 39.24 & 0.18 & 35.45 & 97.93 \\
\hline & & cpa & 31.07 & 0.83 & 29.23 & 0.12 & 32.34 & 93.62 \\
\hline & & cpa & 24.62 & 1.27 & 38.28 & 0.30 & 36.42 & 100.94 \\
\hline & & cpa & 28.64 & 2.84 & 28.39 & 0.23 & 30.64 & 90.83 \\
\hline
\end{tabular}


TABLE 1 - Continued

\begin{tabular}{|c|c|c|c|c|c|c|c|c|}
\hline $\begin{array}{l}\text { Pol. } \\
\text { Sect. }\end{array}$ & $\begin{array}{c}\text { Sample } \\
\text { (Interval in cm) }\end{array}$ & $\begin{array}{c}\text { Material } \\
\text { Analyzed }\end{array}$ & $\mathrm{Cu}$ & $\mathrm{Ni}$ & $\mathrm{Fe}$ & Co & $\mathrm{S}$ & Total \\
\hline \multicolumn{9}{|c|}{ Hole 332B - Continued } \\
\hline & & cpa & 27.21 & 4.08 & 28.48 & 0.17 & 31.42 & 91.39 \\
\hline & & cpa & 24.64 & 4.65 & 26.99 & 0.37 & 31.92 & 90.93 \\
\hline & & po & 0.0 & 0.18 & 59.06 & 0.35 & 38.86 & 98.52 \\
\hline & & po & 0.0 & 0.04 & 61.22 & 0.31 & 40.59 & 102.18 \\
\hline & & po & 0.0 & 0.0 & 61.15 & 0.37 & 41.04 & 102.61 \\
\hline & & po & 0.11 & 0.84 & 60.42 & 0.31 & 40.29 & 102.01 \\
\hline & & po & 0.0 & 0.0 & 60.08 & 0.29 & 39.97 & 100.40 \\
\hline & & poa & 0.0 & 0.90 & 60.67 & 0.42 & 40.91 & 102.90 \\
\hline & & po & 0.01 & 0.0 & 60.32 & 0.32 & 39.73 & 100.43 \\
\hline & & po & 0.0 & 0.16 & 59.61 & 0.44 & 38.60 & 98.85 \\
\hline & & po & 0.0 & 0.31 & 60.91 & 0.34 & 40.59 & 102.23 \\
\hline & & poa & 0.06 & 0.88 & 60.47 & 0.26 & 40.46 & 102.20 \\
\hline & & poa & 0.07 & 0.01 & 61.44 & 0.48 & 40.65 & 102.70 \\
\hline & & po & 0.0 & 0.10 & 58.87 & 0.42 & 39.11 & 98.58 \\
\hline & & pnp & 0.69 & 27.39 & 33.99 & 1.97 & 35.66 & 99.78 \\
\hline & & py & 0.01 & 0.16 & 46.88 & 0.25 & 53.37 & 100.88 \\
\hline & & py & 0.0 & 0.0 & 46.90 & 0.05 & 53.63 & 100.69 \\
\hline \multirow[t]{23}{*}{ DAG-12 } & $44-6,40-42$ & $\mathrm{gb}$ & 0.17 & 0.19 & 63.07 & 0.22 & 31.88 & 95.72 \\
\hline & & $\mathrm{gb}$ & 1.01 & 1.17 & 58.32 & 0.31 & 35.23 & 96.11 \\
\hline & & $\mathrm{gb}$ & 0.11 & 1.17 & 57.53 & 0.40 & 36.43 & 95.71 \\
\hline & & gb & 4.89 & 0.63 & 55.31 & 0.39 & 32.35 & 93.61 \\
\hline & & $\mathrm{gb}$ & 13.74 & 0.91 & 48.34 & 0.36 & 32.68 & 96.15 \\
\hline & & gbp & 0.18 & 0.81 & 57.07 & 0.65 & 39.20 & 98.00 \\
\hline & & gbp & 23.36 & 0.57 & 32.92 & 0.67 & 32.69 & 90.26 \\
\hline & & cpa & 31.11 & 0.12 & 28.90 & 0.05 & 31.65 & 91.97 \\
\hline & & сра & 26.77 & 3.16 & 28.94 & 0.0 & 30.49 & 89.42 \\
\hline & & po & 0.06 & 0.16 & 60.96 & 0.33 & 38.81 & 100.38 \\
\hline & & po & 0.01 & 0.05 & 60.46 & 0.13 & 40.18 & 100.86 \\
\hline & & poa & 0.20 & 0.45 & 60.54 & 0.36 & 39.52 & 101.13 \\
\hline & & poa & 0.12 & 0.97 & 60.39 & 0.19 & 39.71 & 101.58 \\
\hline & & po & 0.09 & 0.69 & 60.14 & 0.40 & 39.43 & 100.86 \\
\hline & & po & 0.10 & 0.18 & 60.65 & 0.44 & 39.99 & 101.35 \\
\hline & & po & 0.10 & 0.17 & 60.88 & 0.21 & 39.79 & 101.24 \\
\hline & & poa & 0.17 & 0.62 & 60.89 & 0.25 & 38.46 & 100.50 \\
\hline & & po & 0.06 & 0.17 & 58.70 & 0.17 & 37.97 & 97.17 \\
\hline & & po & 0.08 & 0.26 & 61.12 & 0.29 & 38.90 & 100.75 \\
\hline & & po & 0.07 & 0.06 & 60.58 & 0.20 & 38.53 & 99.53 \\
\hline & & po & 0.05 & 0.45 & 61.24 & 0.25 & 34.88 & 96.87 \\
\hline & & po & 0.03 & 0.14 & 60.69 & 0.15 & 37.38 & 98.46 \\
\hline & & po & 0.04 & 0.0 & 60.12 & 0.20 & 37.60 & 98.09 \\
\hline \multirow[t]{9}{*}{ DAG-13 } & $45-1,70-72$ & gb & 4.20 & 4.07 & 5278 & 0.13 & 33.70 & 94.91 \\
\hline & & $\mathrm{gb}$ & 1.14 & 1.76 & 57.89 & 0.25 & 35.65 & 96.74 \\
\hline & & gb & 1.92 & 6.68 & 51.86 & 0.25 & 34.66 & 95.42 \\
\hline & & $\mathrm{cp}$ & 34.42 & 0.0 & 27.73 & 0.0 & 34.38 & 96.54 \\
\hline & & $\mathrm{cp}$ & 33.60 & 0.0 & 29.47 & 0.0 & 34.69 & 97.76 \\
\hline & & py & 0.0 & 0.0 & 47.53 & 0.0 & 52.88 & 100.40 \\
\hline & & py & 0.0 & 0.0 & 47.32 & 0.07 & 54.86 & 102.39 \\
\hline & & py & 0.06 & 0.01 & 47.44 & 0.19 & 54.47 & 102.31 \\
\hline & & py & 0.0 & 0.06 & 48.15 & 0.17 & 56.52 & 104.98 \\
\hline \multirow[t]{8}{*}{ DAG-14 } & $46-1,45-47$ & $\mathrm{cp}$ & 24.81 & 1.08 & 39.05 & 0.34 & 35.35 & 101.32 \\
\hline & & $\mathrm{cp}$ & 33.85 & 0.09 & 30.01 & 0.12 & 33.40 & 97.68 \\
\hline & & $\mathrm{cp}$ & 27.60 & 0.65 & 34.53 & 0.35 & 35.19 & 98.51 \\
\hline & & $\mathrm{cp}, \mathrm{py}$ & 25.75 & 3.86 & 28.02 & 1.95 & 32.80 & 92.45 \\
\hline & & py & 0.07 & 0.53 & 47.02 & 1.13 & 51.78 & 100.67 \\
\hline & & py & 0.0 & 0.06 & 45.70 & 0.09 & 52.91 & 98.89 \\
\hline & & py & 0.08 & 0.04 & 46.88 & 0.12 & 53.73 & 100.95 \\
\hline & & py & 0.02 & 0.08 & 46.99 & 0.05 & 52.29 & 99.50 \\
\hline \multirow[t]{11}{*}{ DAG-15 } & $46-1,53-55$ & $\mathrm{gb}$ & 0.37 & 0.35 & 59.20 & 0.09 & 33.00 & 93.17 \\
\hline & & $\mathrm{cp}$ & 29.59 & 0.30 & 30.40 & 0.39 & 35.13 & 95.91 \\
\hline & & $\mathrm{cp}$ & 25.83 & 0.95 & 35.89 & 0.42 & 34.92 & 98.09 \\
\hline & & $\mathrm{cp}, \mathrm{py}$ & 18.69 & 3.84 & 29.79 & 4.29 & 39.05 & 95.80 \\
\hline & & cp & 20.76 & 6.75 & 22.47 & 12.28 & 35.12 & 97.46 \\
\hline & & $\mathrm{cp}$ & 27.99 & 0.52 & 33.74 & 0.38 & 35.20 & 98.02 \\
\hline & & $\mathrm{cp}$ & 28.79 & 0.84 & 34.88 & 2.23 & 31.40 & 103.29 \\
\hline & & py & 0.06 & 0.06 & 27.14 & 0.12 & 54.18 & 101.63 \\
\hline & & py, cp & 2.38 & 0.61 & 43.77 & 0.56 & 51.90 & 99.28 \\
\hline & & py & 0.05 & 0.03 & 47.48 & 0.21 & 53.92 & 101.78 \\
\hline & & py & 0.01 & 0.90 & 45.49 & 1.21 & 52.50 & 100.22 \\
\hline
\end{tabular}


TABLE 1 -Continued

\begin{tabular}{|c|c|c|c|c|c|c|c|c|}
\hline $\begin{array}{l}\text { Pol. } \\
\text { Sect. }\end{array}$ & $\begin{array}{c}\text { Sample } \\
\text { (Interval in } \mathrm{cm} \text { ) }\end{array}$ & $\begin{array}{c}\text { Material } \\
\text { Analyzed } \\
\end{array}$ & $\mathrm{Cu}$ & $\mathrm{Ni}$ & $\mathrm{Fe}$ & Co & $\mathrm{S}$ & Total \\
\hline \multicolumn{9}{|c|}{ Hole 332B - Continued } \\
\hline \multirow[t]{13}{*}{ DAG-16 } & $46-1,75-77$ & $\mathrm{cp}$ & 18.71 & 0.93 & 45.47 & 0.35 & 35.65 & 101.62 \\
\hline & & $\mathrm{cp}$ & 27.28 & 0.65 & 36.35 & 0.24 & 34.90 & 99.78 \\
\hline & & cp & 22.94 & 1.04 & 41.07 & 0.30 & 35.52 & 101.30 \\
\hline & & $\mathrm{cp}$ & 26.34 & 0.62 & 36.87 & 0.18 & 36.18 & 100.74 \\
\hline & & cp & 24.92 & 0.84 & 39.55 & 0.16 & 35.29 & 101.36 \\
\hline & & cp & 19.31 & 1.30 & 44.21 & 0.27 & 35.32 & 101.02 \\
\hline & & $\mathrm{cp}$ & 26.77 & 0.70 & 37.63 & 0.28 & 35.60 & 101.60 \\
\hline & & cp & 27.00 & 0.77 & 36.79 & 0.24 & 36.11 & 101.66 \\
\hline & & cp & 16.50 & 1.81 & 46.13 & 0.15 & 36.79 & 101.97 \\
\hline & & $\mathrm{cp}$ & 24.33 & 0.93 & 39.71 & 0.24 & 37.17 & 102.99 \\
\hline & & py & 0.02 & 0.01 & 49.29 & 0.14 & 53.86 & 103.68 \\
\hline & & py & 0.02 & 0.02 & 49.30 & 0.01 & 55.34 & 105.33 \\
\hline & & py & 0.04 & 0.03 & 48.70 & 0.0 & 53.70 & 103.15 \\
\hline \multirow[t]{14}{*}{ DAG-17 } & $46-2,24-26$ & $\mathrm{gb}$ & 6.28 & 0.38 & 55.92 & 0.15 & 34.38 & 97.69 \\
\hline & & gb & 18.38 & 1.16 & 44.12 & 0.37 & 36.23 & 100.71 \\
\hline & & $\mathrm{gb}$ & 2.26 & 0.49 & 60.21 & 0.23 & 34.90 & 98.62 \\
\hline & & $\mathrm{gb}$ & 4.27 & 0.48 & 55.56 & 0.06 & 37.87 & 98.70 \\
\hline & & $\mathrm{gb}$ & 11.82 & 0.78 & 51.57 & 0.31 & 34.08 & 99.09 \\
\hline & & $\mathrm{cp}$ & 23.60 & 2.00 & 37.63 & 0.39 & 34.93 & 98.70 \\
\hline & & $\mathrm{cp}$ & 32.02 & 0.22 & 29.69 & 0.95 & 33.46 & 96.47 \\
\hline & & $\mathrm{cp}$ & 28.50 & 1.06 & 32.71 & 1.50 & 34.81 & 98.86 \\
\hline & & $\mathrm{cp}$ & 18.17 & 3.01 & 41.28 & 0.46 & 35.79 & 98.89 \\
\hline & & po & 0.0 & 0.03 & 59.51 & 0.26 & 38.25 & 98.36 \\
\hline & & po & 0.0 & 0.0 & 58.47 & 0.31 & 39.11 & 98.15 \\
\hline & & po & 0.0 & 0.0 & 59.46 & 0.25 & 38.81 & 98.78 \\
\hline & & po & 0.0 & 0.01 & 57.96 & 0.26 & 40.03 & 98.59 \\
\hline & & po & 0.0 & 0.0 & 60.11 & 0.27 & 39.93 & 100.68 \\
\hline \multirow[t]{15}{*}{ DAG-18 } & $47-2,110-113$ & $\mathrm{gb}$ & 14.25 & 0.08 & 47.13 & 0.33 & 35.94 & 97.82 \\
\hline & & $\mathrm{gb}$ & 0.0 & 0.0 & 60.86 & 0.10 & 36.35 & 97.34 \\
\hline & & gbp & 11.32 & 0.12 & 50.64 & 0.83 & 32.52 & 95.40 \\
\hline & & $\mathrm{gb}$ & 2.79 & 0.04 & 57.86 & 0.18 & 37.36 & 98.27 \\
\hline & & $\mathrm{gb}$ & 1.16 & 0.07 & 58.64 & 0.21 & 37.08 & 97.18 \\
\hline & & $\mathrm{gb}$ & 2.92 & 0.48 & 58.98 & 0.18 & 32.49 & 95.10 \\
\hline & & $\mathrm{gb}$ & 12.15 & 0.82 & 48.74 & 0.17 & 36.80 & 98.20 \\
\hline & & $\mathrm{gb}$ & 4.41 & 0.19 & 56.69 & 0.18 & 37.18 & 98.69 \\
\hline & & $\mathrm{gb}$ & 12.78 & 1.01 & 48.59 & 0.37 & 36.48 & 99.29 \\
\hline & & gb & 0.16 & 0.0 & 60.14 & 0.17 & 36.31 & 96.82 \\
\hline & & $\mathrm{gb}$ & 24.54 & 0.37 & 38.96 & 0.80 & 34.91 & 99.64 \\
\hline & & $\mathrm{cp}$ & 30.36 & 0.81 & 33.54 & 0.22 & 34.37 & 99.30 \\
\hline & & $\mathrm{cp}$ & 32.00 & 0.54 & 32.15 & 0.22 & 34.48 & 99.39 \\
\hline & & $\mathrm{cp}$ & 20.66 & 2.75 & 41.16 & 0.24 & 35.16 & 99.97 \\
\hline & & py & 0.0 & 0.43 & 46.65 & 0.54 & 50.65 & 98.28 \\
\hline \multirow[t]{9}{*}{ DAG-19 } & $47-2,111$ & $\mathrm{gb}$ & 0.13 & 0.10 & 61.43 & 0.19 & 38.69 & 100.60 \\
\hline & & $\mathrm{gb}$ & 0.06 & 0.09 & 59.49 & 0.43 & 39.09 & 99.22 \\
\hline & & $\mathrm{gb}$ & 4.79 & 2.79 & 55.08 & 0.93 & 35.47 & 99.33 \\
\hline & & $\mathrm{gb}$ & 0.14 & 0.15 & 62.90 & 0.69 & 32.76 & 99.77 \\
\hline & & $\mathrm{cp}, \mathrm{py}$ & 32.84 & 0.49 & 29.69 & 0.43 & 36.56 & 100.01 \\
\hline & & $\mathrm{cp}$ & 29.70 & 0.66 & 31.48 & 0.25 & 34.51 & 96.51 \\
\hline & & py & 0.03 & 0.24 & 48.30 & 0.23 & 52.74 & 101.60 \\
\hline & & py, cp & 0.38 & 0.17 & 46.98 & 0.33 & 53.24 & 101.18 \\
\hline & & py & 0.0 & 0.13 & 48.39 & 0.06 & 54.08 & 102.66 \\
\hline \multirow[t]{12}{*}{ DAG-20 } & $47-3,116-117$ & $\mathrm{gb}$ & 0.13 & 0.0 & 63.57 & 0.0 & 31.47 & 95.22 \\
\hline & & $\mathrm{gbp}$ & 14.50 & 0.89 & 47.64 & 0.0 & 34.29 & 97.32 \\
\hline & & gbp & 0.30 & 0.22 & 63.04 & 0.0 & 33.22 & 96.80 \\
\hline & & $\mathrm{gb}$ & 1.07 & 0.32 & 62.73 & 0.0 & 33.92 & 98.07 \\
\hline & & $\mathrm{gb}$ & 17.68 & 1.78 & 43.95 & 0.58 & 35.50 & 99.54 \\
\hline & & $\mathrm{gb}$ & 7.49 & 0.52 & 54.36 & 0.01 & 36.68 & 99.08 \\
\hline & & cp & 33.99 & 0.0 & 30.05 & 0.05 & 33.34 & 97.93 \\
\hline & & cp & 34.30 & 0.0 & 30.29 & 0.35 & 35.30 & 100.40 \\
\hline & & $c p$ & 31.70 & 0.17 & 32.34 & 0.22 & 36.12 & 101.27 \\
\hline & & $\mathrm{cp}$ & 34.31 & 0.0 & 30.03 & 0.01 & 31.32 & 95.82 \\
\hline & & py & 0.01 & 0.21 & 47.79 & 0.32 & 52.56 & 100.92 \\
\hline & & py & 0.0 & 0.0 & 49.03 & 0.08 & 52.91 & 102.07 \\
\hline \multicolumn{9}{|c|}{ Hole 333A } \\
\hline \multirow[t]{2}{*}{ DAG-21 } & $8-4,44$ & gb & 14.67 & 2.0 & 39.73 & 0.06 & 32.47 & 88.97 \\
\hline & & $\mathrm{gb}$ & 0.0 & 0.0 & 51.32 & 0.0 & 30.28 & 81.60 \\
\hline \multirow[t]{3}{*}{ DAG-22 } & $10-1,13$ & $\mathrm{gb}$ & 1.42 & 0.09 & 54.73 & 0.34 & 31.53 & 88.15 \\
\hline & & $\mathrm{gb}$ & 2.51 & 5.42 & 48.86 & 0.49 & 34.13 & 91.44 \\
\hline & & $\mathrm{cp}$ & 24.86 & 0.0 & 38.93 & 0.30 & 35.77 & 99.96 \\
\hline
\end{tabular}


TABLE 1 - Continued

\begin{tabular}{|c|c|c|c|c|c|c|c|c|}
\hline $\begin{array}{l}\text { Pol. } \\
\text { Sect. }\end{array}$ & $\begin{array}{c}\text { Sample } \\
\text { (Interval in } \mathrm{cm} \text { ) }\end{array}$ & $\begin{array}{r}\text { Material } \\
\text { Analyzed }\end{array}$ & $\mathrm{Cu}$ & $\mathrm{Ni}$ & $\mathrm{Fe}$ & Co & $\mathrm{S}$ & Total \\
\hline \multicolumn{9}{|c|}{ Hole 333A - Continued } \\
\hline & & $\mathrm{cp}$ & 21.17 & 0.91 & 42.39 & 0.23 & 35.36 & 100.06 \\
\hline & & cpp & 21.69 & 1.49 & 40.61 & 0.39 & 34.77 & 98.99 \\
\hline & & cpp? & 10.34 & 3.32 & 50.62 & 0.40 & 35.58 & 100.31 \\
\hline & & $\mathrm{cp}$ & 21.78 & 1.18 & 39.86 & 0.25 & 34.88 & 98.32 \\
\hline & & cp & 23.08 & 0.0 & 39.59 & 0.32 & 35.19 & 98.56 \\
\hline & & py & 0.17 & 0.06 & 47.41 & 0.38 & 52.71 & 100.83 \\
\hline \multirow[t]{13}{*}{ DAG-23 } & $10-10,64$ & $\mathrm{gb}$ & 10.8 & 1.26 & 49.41 & 0.23 & 32.40 & 95.17 \\
\hline & & $\mathrm{gb}$ & 2.42 & 0.30 & 58.07 & 0.25 & 33.24 & 94.80 \\
\hline & & gbp & 13.31 & 3.69 & 44.31 & 0.85 & 35.13 & 97.76 \\
\hline & & gbp & 0.25 & 23.55 & 38.43 & 0.87 & 34.13 & 97.72 \\
\hline & & $\mathrm{gb}$ & 2.53 & 0.58 & 57.43 & 0.36 & 33.06 & 94.40 \\
\hline & & $\mathrm{gb}$ & 0.50 & 0.17 & 59.48 & 0.27 & 32.02 & 92.95 \\
\hline & & $\mathrm{gb}$ & 1.54 & 0.37 & 58.59 & 0.18 & 31.50 & 92.61 \\
\hline & & $\mathrm{gb}$ & 2.11 & 0.12 & 58.34 & 0.16 & 30.51 & 91.80 \\
\hline & & $\mathrm{gb}$ & 2.72 & 0.15 & 57.41 & 0.02 & 34.41 & 95.15 \\
\hline & & $\mathrm{gb}$ & 1.92 & 3.44 & 55.28 & 0.23 & 36.95 & 98.25 \\
\hline & & $\mathrm{cp}$ & 19.83 & 1.59 & 42.41 & 0.38 & 34.50 & 99.15 \\
\hline & & $\mathrm{cp}$ & 23.41 & 0.90 & 35.44 & 0.25 & 34.35 & 94.70 \\
\hline & & $\mathrm{cp}$ & 24.30 & 1.10 & 36.22 & 0.32 & 33.71 & 95.94 \\
\hline \multirow[t]{12}{*}{ DAG-24 } & $10-3,63$ & gbp & 0.17 & 0.22 & 59.65 & 0.18 & 33.26 & 94.15 \\
\hline & & gbp & 10.70 & 0.26 & 49.45 & 0.18 & 33.36 & 94.65 \\
\hline & & $\mathrm{gb}$ & 15.06 & 1.02 & 46.19 & 0.35 & 35.42 & 98.62 \\
\hline & & $\mathrm{gb}$ & 5.39 & 0.31 & 55.39 & 0.33 & 31.66 & 93.72 \\
\hline & & $\mathrm{gb}$ & 1.92 & 0.24 & 59.21 & 0.40 & 33.91 & 96.19 \\
\hline & & $\mathrm{gb}$ & 1.38 & 1.85 & 57.07 & 0.30 & 37.40 & 98.44 \\
\hline & & $\mathrm{cp}$ & 19.00 & 1.30 & 43.41 & 0.28 & 36.17 & 100.57 \\
\hline & & $\mathrm{cp}$ & 20.80 & 1.04 & 41.12 & 0.29 & 35.17 & 98.95 \\
\hline & & $\mathrm{cp}$ & 24.17 & 0.69 & 37.37 & 0.34 & 35.00 & 98.10 \\
\hline & & $\mathrm{cp}$ & 17.63 & 1.39 & 43.90 & 0.35 & 35.53 & 98.37 \\
\hline & & $\mathrm{cp}$ & 21.29 & 1.04 & 40.76 & 0.31 & 35.07 & 99.07 \\
\hline & & py & 0.0 & 0.60 & 47.22 & 0.10 & 53.32 & 101.61 \\
\hline \multirow[t]{6}{*}{ DAG-25 } & $10-3,75$ & gbp & 0.16 & 3.29 & 57.82 & 0.25 & 38.00 & 99.57 \\
\hline & & gbp & 0.32 & 30.82 & 32.48 & 0.25 & 32.67 & 96.61 \\
\hline & & $\mathrm{gb}$ & 0.16 & 6.85 & 53.91 & 0.47 & 38.10 & 99.59 \\
\hline & & $\mathrm{gb}$ & 6.66 & 14.45 & 41.58 & 0.47 & 35.56 & 98.82 \\
\hline & & $\mathrm{gb}$ & 5.40 & 2.95 & 51.39 & 0.17 & 37.08 & 97.14 \\
\hline & & $\mathrm{gb}$ & 8.06 & 0.26 & 52.72 & 0.16 & 36.18 & 97.52 \\
\hline \multirow[t]{4}{*}{ DAG-25 } & $10-3,75$ & $\mathrm{cp}$ & 18.35 & 2.61 & 43.55 & 0.22 & 36.42 & 101.22 \\
\hline & & $\mathrm{cp}$ & 27.97 & 4.81 & 30.40 & 2.50 & 36.15 & 101.90 \\
\hline & & po & 0.04 & 0.34 & 59.89 & 0.24 & 40.43 & 100.98 \\
\hline & & po & 0.01 & 0.05 & 60.15 & 0.17 & 41.02 & 101.44 \\
\hline \multicolumn{9}{|l|}{ Hole 334} \\
\hline \multirow[t]{23}{*}{ DAG-26 } & $22-2,75-77$ & pnp & 0.17 & 33.34 & 31.20 & 2.16 & 34.23 & 101.45 \\
\hline & & p? & 11.47 & 23.67 & 30.25 & 2.12 & 34.16 & 102.00 \\
\hline & & pn & 0.0 & 32.35 & 29.95 & 2.77 & 33.34 & 98.73 \\
\hline & & pn & 0.0 & 32.92 & 31.71 & 1.58 & 33.50 & 99.50 \\
\hline & & pn & 0.0 & 31.05 & 22.42 & 19.30 & 33.79 & 106.87 \\
\hline & & pnp & 0.0 & 31.39 & 33.02 & 3.17 & 32.71 & 100.82 \\
\hline & & $\mathrm{np}$ & 59.39 & 0.24 & 13.88 & 0.0 & 26.89 & 100.67 \\
\hline & & pnp & 0.10 & 32.92 & 31.47 & 2.38 & 33.34 & 100.61 \\
\hline & & $\mathrm{p}$ ? & 10.25 & 23.30 & 31.04 & 2.34 & 34.07 & 101.39 \\
\hline & & pnp & 2.44 & 30.48 & 31.67 & 3.25 & 34.07 & 102.44 \\
\hline & & p? & 33.38 & 0.08 & 31.22 & 0.0 & 34.99 & 100.10 \\
\hline & & pnp & 0.0 & 31.63 & 33.77 & 2.88 & 34.61 & 103.27 \\
\hline & & p? & 8.50 & 24.08 & 32.28 & 2.82 & 34.48 & 102.54 \\
\hline & & pn & 0.0 & 33.22 & 32.40 & 1.93 & 34.50 & 102.33 \\
\hline & & pnp & 0.73 & 31.13 & 33.52 & 2.14 & 34.22 & 102.07 \\
\hline & & p? & 18.79 & 14.74 & 31.43 & 2.09 & 35.34 & 102.73 \\
\hline & & pn & 0.0 & 33.58 & 32.18 & 1.44 & 34.33 & 101.84 \\
\hline & & pn & 0.0 & 32.44 & 32.84 & 1.58 & 33.89 & 101.85 \\
\hline & & pn & 0.01 & 32.29 & 33.65 & 2.35 & 35.69 & 104.35 \\
\hline & & pnp & 0.62 & 33.18 & 31.56 & 3.71 & 34.84 & 103.23 \\
\hline & & p? & 19.24 & 16.30 & 30.73 & 2.65 & 36.16 & 105.40 \\
\hline & & $\mathrm{cp}$ & 33.39 & 0.26 & 29.83 & 0.0 & 33.81 & 97.72 \\
\hline & & cp & 33.71 & 0.0 & 30.36 & 0.0 & 35.02 & 99.43 \\
\hline \multirow[t]{3}{*}{ DAG-27 } & $23-1,113-119$ & $\mathrm{cp}$ & 33.87 & 0.0 & 27.68 & 0.0 & 33.56 & 95.13 \\
\hline & & $\mathrm{cp}, \mathrm{py}$ & 30.71 & 3.83 & 27.27 & 0.09 & 36.61 & 98.88 \\
\hline & & cpp & 31.26 & 0.39 & 29.89 & 0.21 & 34.15 & 96.20 \\
\hline
\end{tabular}


TABLE 1 - Continued

\begin{tabular}{|c|c|c|c|c|c|c|c|c|}
\hline $\begin{array}{l}\text { Pol. } \\
\text { Sect. }\end{array}$ & $\begin{array}{c}\text { Sample } \\
\text { (Interval in } \mathrm{cm} \text { ) }\end{array}$ & $\begin{array}{c}\text { Material } \\
\text { Analyzed }\end{array}$ & $\mathrm{Cu}$ & $\mathrm{Ni}$ & $\mathrm{Fe}$ & $\mathrm{Co}$ & $\mathrm{S}$ & Total \\
\hline \multirow[t]{19}{*}{ Hole 334} & - Continued & & & & & & & \\
\hline & & po & 0.09 & 1.53 & 57.96 & 0.22 & 37.60 & 97.79 \\
\hline & & pop & 0.08 & 0.03 & 58.67 & 0.15 & 37.03 & 97.26 \\
\hline & & $\mathrm{p}$ ? & 25.37 & 8.86 & 27.89 & 0.19 & 31.44 & 94.30 \\
\hline & & po & 0.71 & 0.61 & 56.77 & 0.11 & 3690 & 95.62 \\
\hline & & pop & 0.16 & 0.10 & 60.37 & 0.20 & 38.12 & 99.53 \\
\hline & & $\mathrm{p}$ ? & 29.19 & 3.05 & 29.86 & 0.23 & 34.08 & 97.04 \\
\hline & & po & 0.04 & 0.76 & 58.90 & 0.23 & 38.36 & 98.84 \\
\hline & & po & 0.05 & 0.47 & 60.43 & 0.39 & 37.78 & 99.65 \\
\hline & & pop & 0.10 & 0.36 & 60.63 & 0.21 & 38.76 & 100.48 \\
\hline & & p? & 25.89 & 8.88 & 29.90 & 0.24 & 32.32 & 97.68 \\
\hline & & po & 0.06 & 0.51 & 61.90 & 0.07 & 38.18 & 101.06 \\
\hline & & po & 0.66 & 3.62 & 53.74 & 0.50 & 36.48 & 95.20 \\
\hline & & pop & 0.16 & 1.36 & 58.02 & 0.13 & 37.58 & 97.48 \\
\hline & & pnp & 0.19 & 29.65 & 29.32 & 0.13 & 32.15 & 91.68 \\
\hline & & pop & 0.11 & 0.52 & 61.07 & 0.13 & 39.11 & 101.33 \\
\hline & & p? & 7.33 & 25.52 & 28.86 & 0.17 & 32.57 & 94.86 \\
\hline & & py & 0.39 & 0.66 & 44.95 & 0.17 & 53.39 & 99.71 \\
\hline & & $\mathrm{py}, \mathrm{cp}$ & 0.24 & 1.43 & 44.43 & 0.13 & 54.37 & 100.95 \\
\hline
\end{tabular}

Note: $\mathrm{g}=$ chalcopyrite - cubanite $\mathrm{cp}=$ pyrrhotite grains $; \mathrm{po}=$ pentlandite grains bn $=$ bornite; py $=$ pyrite grains $; \mathrm{p}=$ phase determined within grain or globule; $\mathrm{p}$ ? = undetermined or possible phase $;$ poa $=$ pyrrhotite with adjacent chalcopyrite $; \mathrm{cpa}=$ chalcopyrite and adjacent pyrrhotite; $\mathrm{cp}, \mathrm{py}=$ chalcopyrite adjacent to pyrite $; \mathrm{py}, \mathrm{cp}=$ pyrite adjacent to chalcopyrite.
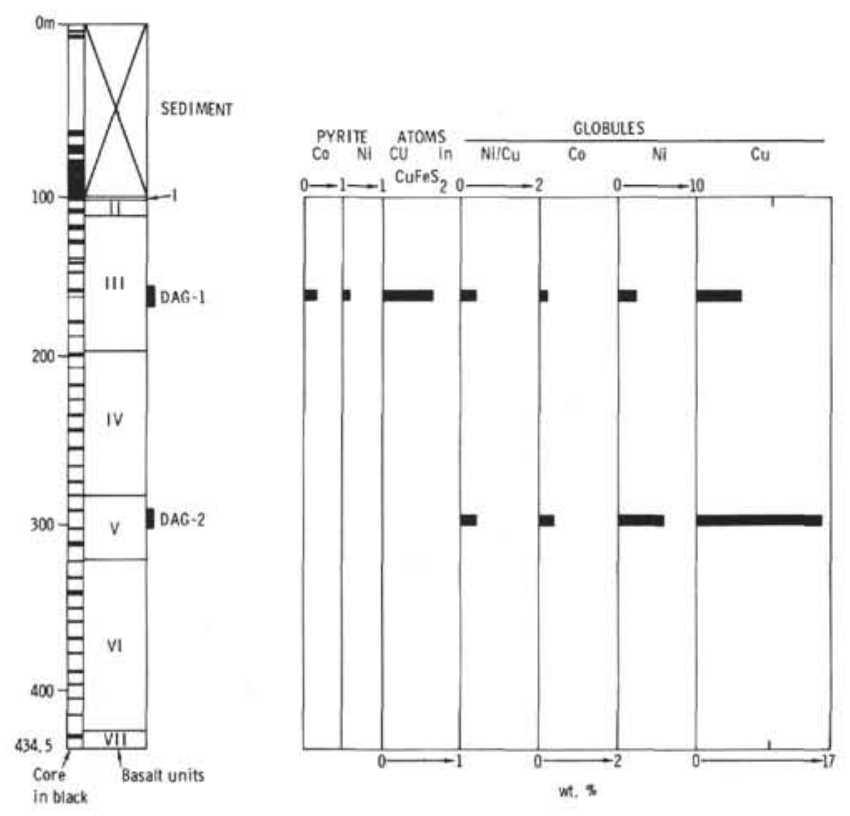

Figure 1. Composition of sulfides in Hole $332 \mathrm{~A}$.

equilibrium was attained throughout the sample below $610^{\circ} \mathrm{C}$, the upper stability limit of pentlandite. The composition of the pentlandite indicates it would be compatible with either pyrrhotite or pyrrhotite and pyrite, depending on temperature, but neither mineral was present.

\section{Late Pyrite Mineralization}

Pyrite on fracture surfaces, in veinlets, and in vesicles in basalt has a very close spatial distribution with zones of alteration in which smectite and calcite are visible. It makes up about $0.5 \%$ of the rock as large euhedral

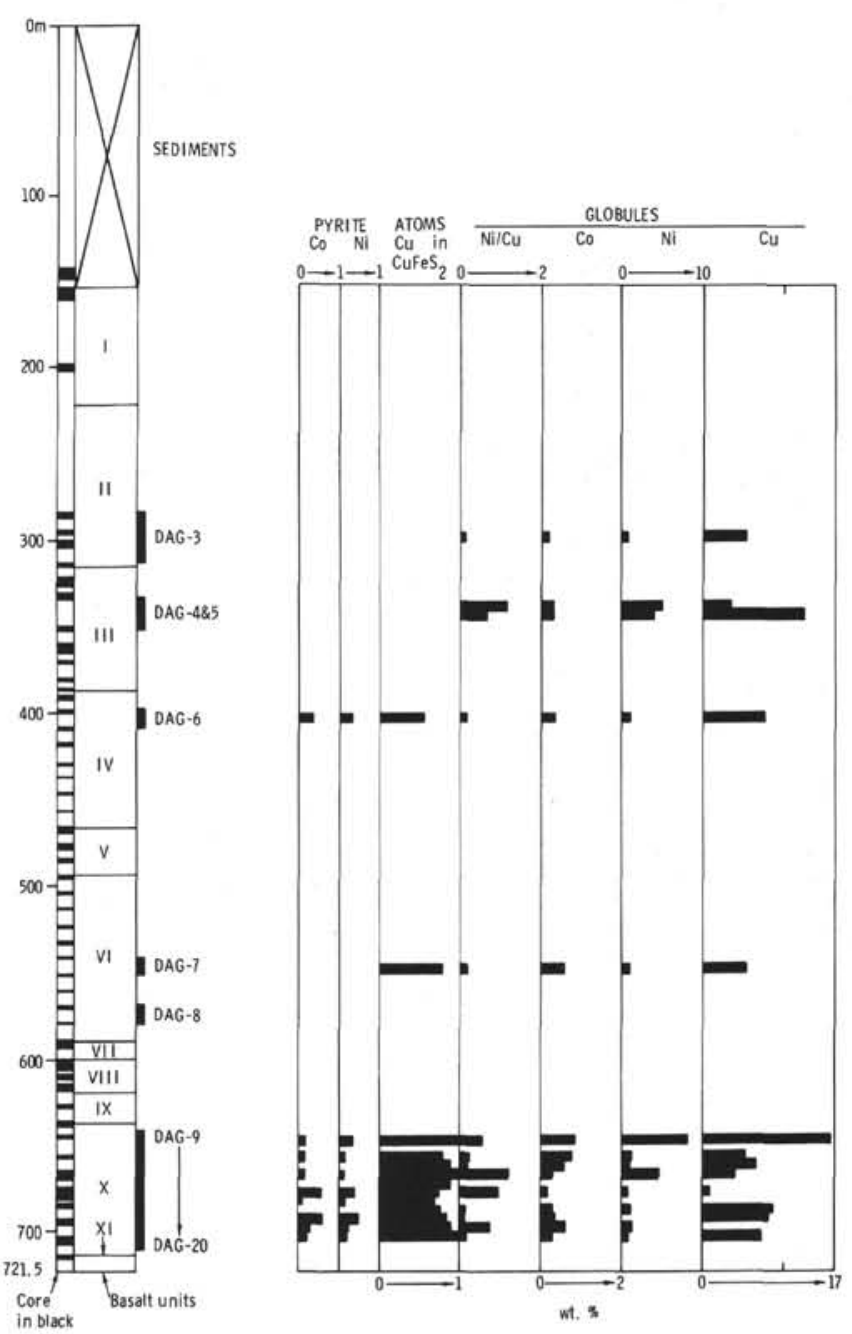

Figure 2. Composition of sulfides in Hole 332B. 

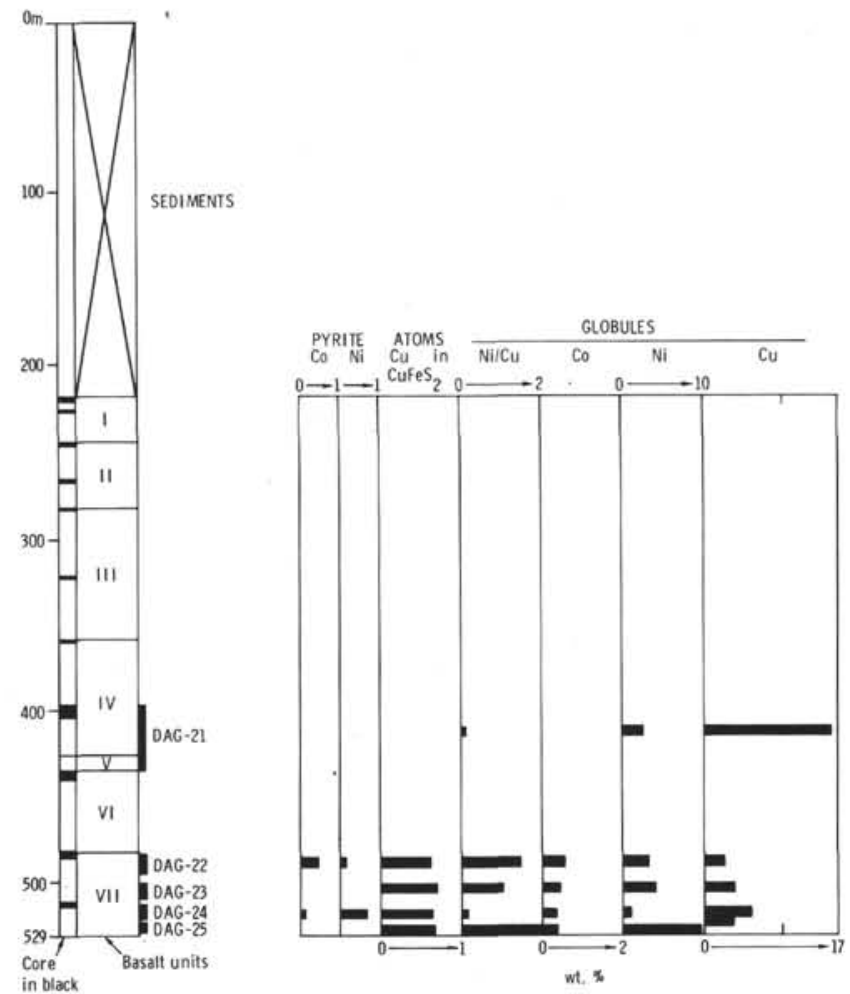

Figure 3. Composition of sulfides in Hole $333 \mathrm{~A}$.

crystals (up to $2 \mathrm{~mm}$ ) and very thin coatings on the surfaces of fractures and vesicles. Small pyrite grains are disseminated through altered basalt. In these zones magmatic globules and pyrrhotite grains are absent but chalcopyrite may be present.

$\mathrm{Co}$ and $\mathrm{Ni}$ range from 0.05 to $0.6 \mathrm{wt} \%$ in pyrite. The distribution of both elements is very erratic both within and between grains. $\mathrm{Mn}, \mathrm{Cu}, \mathrm{Zn}$, and $\mathrm{As}$ were below detection limits.

Clearly the large amounts of $\mathrm{S}, \mathrm{Fe}, \mathrm{Co}$, and $\mathrm{Ni}$ in the pyrite cannot be accounted for by alteration of earlier formed sulfide globules and individual sulfide grains. Sulfur appears to have been added, but $\mathrm{Fe}, \mathrm{Co}$, and $\mathrm{Ni}$ could be derived directly from the basalt.

\section{ACKNOWLEDGMENTS}

I am very grateful to J. Krajewski, research assistant, who did the microprobe analysis and the diligent but tedious search for sulfides in the samples. The research was supported by the National Research Council through Grant NRC-

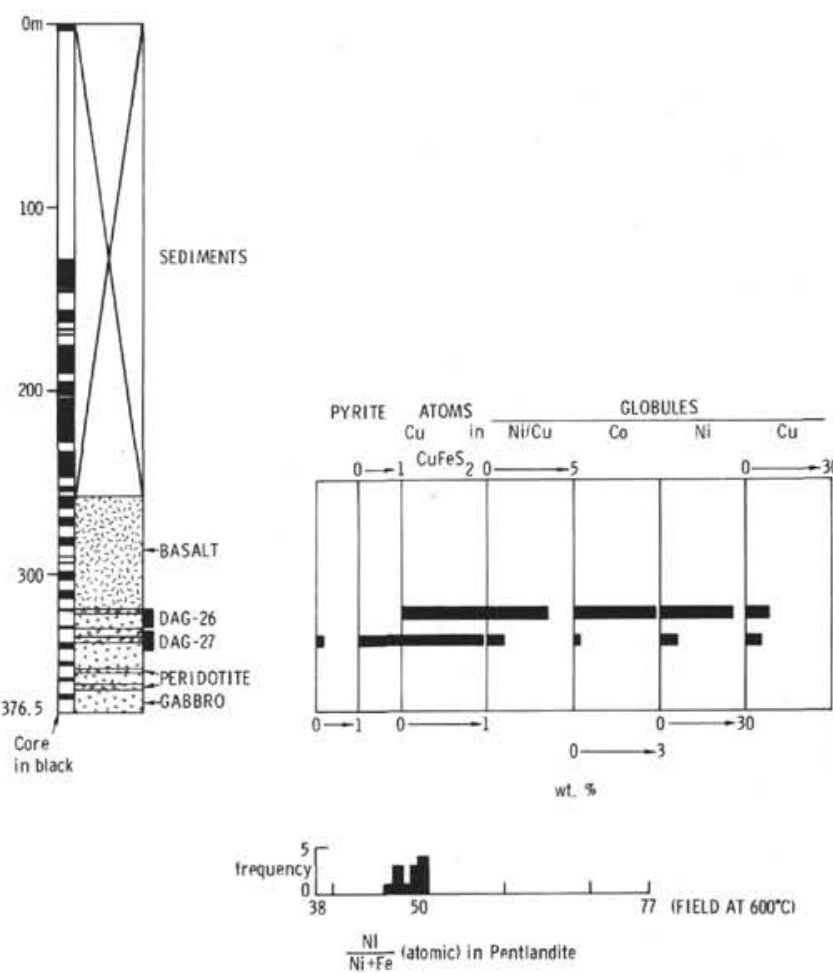

Figure 4. Composition of sulfides at Site 334.

DAG-14. I also wish to thank Dr. J.S. Stevenson for the use of the electron microprobe.

\section{REFERENCES}

Cabri, L.J., 1973. New data on phase relations in the Cu-Fe-S system: Econ. Geol., v. 68, p. 443-454.

Desborough, G.A., Anderson, A.T., and Wright, T.L., 1968. Mineralogy of sulfides from certain Hawaiian basalts: Econ. Geol., v. 63, p. 636-644.

MacLean, W.H. and Shimazaki, H., in preparation. The partition of $\mathrm{Fe}, \mathrm{Co}, \mathrm{Ni}, \mathrm{Cu}$ and $\mathrm{Zn}$ between iron sulfide and iron silicate liquids.

Naldrett, A.J., 1973. Nickel sulfide deposits-their classification and genesis, with special emphasis on deposits of volcanic association: CIMM Bull., v. 66, p. 45-63.

Prinz, M., 1967. Geochemistry of basaltic rocks: trace elements. In Hess, H.H. and Poldervaart, A. (Eds.), Basalts: New York (John Wiley and Sons), p. 271-324.

Skinner, B.J. and Peck, D.L., 1969. An immiscible sulfide melt from Hawaii: In Wilson, H.D.B. (Ed.), Magmatic ore deposits, a symposium: Econ. Geol., Mono. 4, p. 310-322. 